\title{
Frequência de parasitas gastrointestinais em cães e gatos do município de Londrina, PR, com enfoque em saúde pública
}

\section{Frequency of gastrointestinal parasites in dogs and cats of Londrina, PR, focusing on public health}

\author{
Fernanda Pinto Ferreira ${ }^{1 *}$; Renata Cristina Ferreira Dias²; Thais Agostinho Martins ${ }^{3}$; \\ Caroline Constantino ${ }^{4}$; Aline Kuhn Sbruzzi Pasquali ${ }^{5}$; Odilon Vidotto ${ }^{6}$; \\ Roberta Lemos Freire ${ }^{6}$; Italmar Teodorico Navarro ${ }^{6}$
}

\begin{abstract}
Resumo
O objetivo deste trabalho foi determinar a frequência de parasitos gastrointestinais em cães e gatos do município de Londrina, Paraná. Foi realizado um levantamento dos resultados de exames coproparasitológicos, pela técnica de Faust, Willis, Hoffmann e/ou exame direto, executados na rotina do Laboratório de Parasitologia do Departamento de Medicina Veterinária Preventiva da Universidade Estadual de Londrina no período de Janeiro de 2000 a Dezembro de 2011 e no Laboratório de Análises Clínicas Veterinárias de Janeiro de 2008 a Dezembro de 2011. No período foram analisadas 2.668 amostras fecais, sendo $2.290(85,83 \%)$ de cães e $378(14,17 \%)$ de gatos. Deste total, 851(37,16\%) amostras caninas e $166(43,91 \%)$ felinas foram positivas para pelo menos um parasito. Isospora spp. foi o mais frequente em ambas as espécies com $8,82 \%$ dos cães e $11,64 \%$ dos gatos parasitados. Quanto a infestação, 740 (86,96\%) cães e 139 (83,73\%) gatos apresentaram infestação única, enquanto que 111 $(13,04 \%)$ cães e $27(16,27 \%)$ gatos apresentaram múltipla infestação por parasitos gastrointestinais. A ocorrência de um número significativo de parasitos e o estreito contato entre os animais e o homem mostra a necessidade de um controle mais efetivo e específico, visto que a redução da carga parasitária dos animais e, consequentemente do ambiente, diminui a exposição dos humanos a importantes zoonoses.
\end{abstract}

Palavras-chave: Isospora, Giardia, helmintos, protozoários

\begin{abstract}
The aim of this study was to determine the frequency of gastrointestinal parasites in dogs and cats of Londrina, Paraná. A survey of the results of fecal examinations, by the technique Faust, Willis, Hoffmann and / or direct examination, performed in routine Laboratory of Parasitology, Department of Veterinary Preventive Medicine, State University of Londrina in the period January 2000 to December 2011 and the Clinical Laboratory Veterinary January 2008 to December 2011. Were analyzed 2668 fecal samples, of which $2290(85.83 \%)$ of dogs and 378 (14.17\%) of cats. Of the total, $851(37,16 \%)$ dogs
\end{abstract}

\footnotetext{
${ }^{1}$ Pós Graduanda em Zoonoses e Saúde Pública, Dept ${ }^{\circ}$ de Medicina Veterinária Preventiva, DMVP, Universidade Estadual de Londrina, UEL, Londrina, PR. E-mail: nandaferreiravet@gmail.com

2 Discente do Curso de Doutorado em Ciência Animal, DMVP, UEL, Londrina, PR. E-mail: rcfdias14@gmail.com

${ }^{3}$ Pós graduanda em Parasitologia Animal, DMVP, UEL, Londrina, PR. E-mail: thaismedvet620@gmail.com

${ }^{4}$ Discente do Curso de Medicina Veterinária, UEL, Londrina, PR. E-mail: caroline_const@hotmail.com

${ }^{5}$ Discente do Curso de Mestrado em Ciência Animal, DMVP, UEL, Londrina, PR. E-mail alinesbruzzi@gmail.com

${ }^{6}$ Profs. Drs. do Dept ${ }^{\circ}$ de Medicina Veterinária Preventiva, UEL, Londrina, PR. E-mail: vidotto@uel.br; rlfreire@uel.br; italmar@ uel.br

* Autor para correspondência
} 
and $166(43,91 \%)$ feline samples were positive for at least one parasite. Isospora spp. was the most frequent in both species with $8.82 \%$ of infected dogs and $11.64 \%$ of infected cats. Regarding the form of infection, $740(86.96 \%)$ dogs and $139(83.73 \%)$ cats had single infection, while $111(13.04 \%)$ dogs and $27(16.27 \%)$ cats had multiple infection gastrointestinal parasites. The ocurrence of a significant number of parasites and the close contact between animals and humans demonstrates the need for a more effective and specific control, whereas reducing animal load and consequently of the environment, decreases human exposure to important zoonoses.

Key words: Isospora, Giardia, helminths, protozoan

\section{Introdução}

Define-se por zoonoses as doenças naturalmente transmissíveis entre os animais e os seres humanos, com a participação de animais domésticos e selvagens. $\mathrm{O}$ homem pode se infectar pelo contato direto com os animais infectados, ou ainda de forma indireta, entre as quais a ingestão de água e alimentos contaminados estão entre as mais frequentes (LANGONI, 2004). As zoonoses parasitárias, na maioria das vezes, não são fatais em humanos, no entanto, podem causar alergias, diarreias, anemias, além de gastos com diagnóstico e tratamento (SCHANTZ, 1991).

A transmissão é facilitada, muitas vezes, pelos hábitos e costumes indevidos do homem. Xavier (2006) em um questionário realizado com 73 proprietários de 101 cães, em Pelotas, RS, sobre os locais habituais de defecação, observou que $66,4 \%$ dos cães defecam em pátio com grama ou terra, $9,9 \%$ na rua, 9,9\% no piso dentro de casa, $6,9 \%$ em pátio calçado e $6,9 \%$ em jornais dentro da residência. Quanto ao recolhimento de fezes constatou-se que, $82,2 \%$ tinham suas fezes recolhidas do local de defecação diariamente. Nesse mesmo trabalho, foi evidenciado que $65 \%$ dos cães que passeiam regularmente não têm suas fezes recolhidas. Isso demonstra a alta carga parasitária desprezada diariamente no meio ambiente e têm, como agravante, o risco para transmissão de zoonoses.

Inquéritos sobre a prevalência de enteroparasitos têm sido realizados em várias cidades do Brasil, sendo descrita uma maior frequência de Isospora spp., Giardia spp., Ancylostoma spp., Toxocara spp., Platynosomum fastosum, Trichuris spp., Strongyloides stercoralis, Dipylidium caninum,
Physaloptera praeputalis, Trichomonas spp., Cryptospporidium spp., Entamoeba spp., além de ovos de Capilariidae e Taeniidae (VASCONCELLOS; BARROS; OLIVEIRA, 2006; XAVIER, 2006; FUNADA et al., 2007; LEITE et al., 2007; SILVA et al., 2007; FRANCISCO et al., 2008; SILVA et al., 2008; TORRICO et al., 2008).

É constatado que o convívio de pessoas e animais resulta em benefícios significativos, tais como redução da pressão sanguínea e da ocorrência de doenças cardiovasculares (ROBERTSON et al., 2000). Os animais domésticos são utilizados em métodos terapêuticos, como a Terapia Assistida por Animais (TAA) em que cães, gatos e equinos, promovem uma melhora social, emocional, física e cognitiva ao homem (MACHADO et al., 2008).

$\mathrm{O}$ estreito relacionamento entre os animais de estimação e os humanos, principalmente crianças e idosos, que são mais suscetíveis, facilita a cadeia de transmissão das zoonoses. Os animais parasitados são uma fonte para a contaminação do meio ambiente, representando um risco à saúde humana e à de outros animais (OLIVEIRA et al., 2009; CAMPOS FILHO et al., 2008). Dentro desta expectativa de riscos, o trabalho objetivou avaliar a frequência de parasitos gastrointestinais em cães e gatos, a partir de amostras de fezes encaminhadas a dois laboratórios da cidade de Londrina, Paraná.

\section{Material e Métodos}

A avaliação da frequência de parasitos gastrointestinais foi realizada por meio do levantamento dos laudos de exames 
coproparasitológicos de cães e gatos, efetuados no Laboratório de Parasitologia do Departamento de Medicina Veterinária Preventiva (DMVP) da Universidade Estadual de Londrina (UEL), no período de 2000 a 2011, e no Laboratório de Análises Clínicas Veterinárias (ANCLIVET), entre os anos de 2008 a 2011.

Os métodos de diagnósticos empregados na rotina dos laboratórios foram: exame direto em fezes recém-coletadas (HOFFMANN, 1987), método de Willis (1921), método de Faust et al. (1939) e o método de Hoffmann, Pons e Janer (1934).

Dados de idade, sexo e raça dos animais foram colhidos e tabulados, bem como os resultados dos exames coproparasitológicos. Calculou-se a frequência dos resultados e aplicou-se o teste de duas proporções, qui-quadrado, utilizando-se o nível de significância de 5\%.

\section{Resultados}

No Laboratório de Parasitologia do DMVP-UEL foram realizados 2.273 exames coproparasitológicos e, no ANCLIVET, 395. Das 2.668 amostras fecais, $2.290(85,83 \%)$ foram de cães e $378(14,17 \%)$ de gatos. Das 2.290 amostras de fezes de cães, 851 $(37,16 \%)$ foram positivas para um ou mais parasito; e das 378 amostras de fezes de gatos, 166 (43,91\%) foram positivas.

A distribuição dos animais parasitados em relação às variáveis idade, sexo e raça estão descritos na Tabela 1. A faixa etária variou de 20 dias a 21 anos sendo que a mais prevalente, tanto para os cães quanto para os gatos parasitados, encontrava-se entre um mês e um ano de idade $(p<0,05)$. Quanto ao sexo, 409 (50,49\%) amostras positivas de cães eram de fêmeas e $401(49,51 \%)$ de machos ( $p>0,05)$; entre os gatos $50(45,05 \%)$ eram fêmeas e 61 $(54,95 \%)$ machos ( $p>0,05)$. Quando comparadas as proporções para a variável raça, entre os cães e entre os gatos parasitados, verificou-se maior prevalência $(p<0,05)$ em cães sem raça $(60,62)$ e em gatos com raça $(70,71 \%)$. As diferenças numéricas quanto ao sexo, idade e raça deve-se à falta de informações nas requisições de exames.

Tabela 1. Distribuição de amostras fecais de cães e de gatos parasitados, segundo a idade, sexo e raça, em dois laboratórios de parasitologia veterinária, Londrina-PR, no período de 2000 a 2011.

\begin{tabular}{|c|c|c|}
\hline \multirow{2}{*}{ Variáveis } & Cães & Gatos \\
\hline & Positivos (\%) & Positivos (\%) \\
\hline \multicolumn{3}{|l|}{ Idade } \\
\hline$<1$ mês & $35(4,85)$ & $3(2,65)$ \\
\hline 1 mês -| 1 ano & $478(66,20)$ & $72(63,75)$ \\
\hline 1ano -5 anos & $129(17,87)$ & $26(23,01)$ \\
\hline$>5$ anos & $80(11,08)$ & $12(10,62)$ \\
\hline Total & $722(100,00)$ & $113(100,00)$ \\
\hline \multicolumn{3}{|l|}{ Sexo } \\
\hline Fêmea & $409(50,49)$ & $50(45,05)$ \\
\hline Macho & $401(49,51)$ & $61(54,95)$ \\
\hline Total & $810(100,00)$ & $111(100,00)$ \\
\hline \multicolumn{3}{|l|}{ Raça } \\
\hline Com Raça & $319(39,38)$ & $99(70,71)$ \\
\hline Sem Raça & $491(60,62)$ & $41(29,29)$ \\
\hline Total & $810(100,00)$ & $140(100,00)$ \\
\hline
\end{tabular}

Fonte: Elaboração dos autores.

Na Tabela 2 está exposto, em ordem decrescente, os parasitos mais prevalentes dentre os animais positivos $(n=851, n=166)$ e dentre o total de animais analisados $(n=2290, n=378)$. 
Tabela 2. Frequência de parasitos em amostras fecais de cães e gatos, em dois laboratórios de parasitologia veterinária, Londrina-PR, no período de 2000 a 2011.

\begin{tabular}{lccc}
\hline & Frequência & $(\%)$ & $(\%)$ \\
\hline Cães & & (Positivos $=\mathbf{8 5 1})$ & (Total = 2290) \\
Isospora spp & 202 & 23,74 & 8,82 \\
Giardia spp & 198 & 23,27 & 8,65 \\
Ancylostoma spp & 149 & 17,51 & 6,51 \\
Toxocara spp & 138 & 16,22 & 6,03 \\
Entamoeba spp & 95 & 11,16 & 4,15 \\
Trichuris vulpis & 35 & 4,11 & 1,53 \\
Outros* & 34 & 4,00 & 1,48 \\
Total & $\mathbf{8 5 1}$ & $\mathbf{1 0 0}$ & $\mathbf{3 7 , 1 6}$ \\
\hline Gatos & & (Positivos $=\mathbf{1 6 6})$ & $\mathbf{( T o t a l}=\mathbf{3 7 8})$ \\
Isospora spp & 44 & 26,51 & 11,64 \\
Giardia spp & 41 & 24,70 & 10,85 \\
Ancylostoma spp & 35 & 21,08 & 9,26 \\
Entamoeba spp & 18 & 10,84 & 4,76 \\
Platynosomum fastosum & 9 & 5,42 & 2,38 \\
Dipylidium caninum & 4 & 2,41 & 1,06 \\
Toxoplasma gondii & 4 & 2,41 & 1,06 \\
Physolaptera praeputialis & 4 & 2,41 & 1,06 \\
Toxocara spp & 3 & 1,81 & 0,79 \\
Outros** & 4 & 2,41 & 1,06 \\
Total & $\mathbf{1 6 6}$ & $\mathbf{1 0 0}$ & $\mathbf{4 3 , 9 1}$ \\
\hline
\end{tabular}

*Trichomonas spp, Dipylidium caninum, Sarcocystis spp, Taenia spp, Uncinaria spp e Echinococcus spp.

**Trichomonas spp, Endolimax nana, Taenia spp e Hammondia hammondi

Fonte: Elaboração dos autores.

Dos animais parasitados, $740(86,96 \%)$ cães e $139(83,73 \%)$ gatos exibiram infestação única enquanto que $111(13,04 \%)$ cães e $27(16,27 \%)$ gatos apresentaram mais de uma infestação por parasitos, principalmente, por Isospora spp e Giardia spp (Tabela 3).

\section{Discussão}

Este levantamento foi realizado com o intuito de mostrar a frequência dos principais endoparasitos encontrados em amostras de cães analisadas no Laboratório de Parasitologia do DMVP-UEL e ANCLIVET

Cystoisospora spp, observado com uma frequência de $8,82 \%$ em cães e $11,64 \%$ em gatos, pode infectar animais domésticos, silvestres e o homem. Esses protozoários são organismos unicelulares que provocam uma infestação na parede intestinal destruindo o epitélio. O animal elimina o oocisto com as fezes, podendo contaminar tanto o solo quanto à água. A isosporose humana é uma zoonose com grande importância em pacientes imunocomprometidos, tais como portadores do vírus HIV, idosos e crianças, pois apresenta manifestações clínicas diretamente ligadas ao sistema imunológico, há um quadro diarreico intenso, com severa desidratação, evoluindo ou não para uma infestação crônica com síndrome de má absorção intestinal. (URQUHART et al., 1998; VASCONCELOS et al., 2008; PEREIRA et al., 2009). Resultados semelhantes foram observados por Dimas, Sebadelhe e Rodrigues (2004) no Município de São Vicente-SP, onde examinaram fezes de 150 cães e observaram uma positividade de 9,72\% para Cystoisospora spp. Tesserolli, 
Fayzano e Agottani (2005) com objetivo de listar as parasitoses de maior importância em Curitiba, no
Estado do Paraná, verificaram uma prevalência de $10 \%$ nos 30 gatos investigados.

Tabela 3. Frequência de infestação múltipla de parasitas, dentre as fezes analisadas na rotina de dois laboratórios de parasitologia veterinária, Londrina-PR, no período de 2000 a 2011.

\begin{tabular}{|c|c|c|c|c|}
\hline & \multicolumn{2}{|c|}{$\begin{array}{c}\text { Cães } \\
(\mathrm{n}=851)\end{array}$} & \multicolumn{2}{|c|}{$\begin{array}{l}\text { Gatos } \\
(\mathrm{n}=166)\end{array}$} \\
\hline & Positivos & $(\%)$ & Positivos & $(\%)$ \\
\hline \multicolumn{5}{|l|}{ Parasitos intestinais } \\
\hline Isospora spp e Giardia spp & 18 & 0,021 & 9 & 0,054 \\
\hline Ancylostoma spp e Toxocara spp & 17 & 0,020 & 1 & 0,006 \\
\hline Ancylostoma spp e Isospora spp & 11 & 0,013 & 3 & 0,018 \\
\hline Entamoeba spp e Isospora spp & 8 & 0,009 & 2 & 0,012 \\
\hline Ancylostoma spp e Trichuris vulpi & 7 & 0,008 & - & - \\
\hline Giardia spp e Entamoeba spp & 5 & 0,006 & 2 & 0,012 \\
\hline Toxocara spp e Isospora spp & 5 & 0,006 & - & - \\
\hline Giardia spp e Toxocara spp & 4 & 0,005 & - & - \\
\hline Ancylostoma spp e Giardia spp & 4 & 0,005 & - & - \\
\hline Toxocara spp e Trichuris vulpis & 3 & 0,004 & - & - \\
\hline Entamoeba spp e Trichuris vulpis & 3 & 0,004 & - & - \\
\hline Entamoeba spp e Trichomonas spp & 3 & 0,004 & 1 & 0,006 \\
\hline Toxocara spp e Entamoeba spp & 2 & 0,002 & - & - \\
\hline Toxocara spp e Dipylidium caninum & 1 & 0,001 & - & - \\
\hline Ancylostoma spp e Entamoeba spp & 1 & 0,001 & 2 & 0,012 \\
\hline Giardia spp e Dipylidium caninum & 1 & 0,001 & 1 & 0,006 \\
\hline Giardia spp e Isospora spp & 1 & 0,001 & - & - \\
\hline Trichomonas spp e Giardia spp & 1 & 0,001 & - & - \\
\hline Toxocara spp e Trichomonas spp & 1 & 0,001 & - & - \\
\hline Physaloptera praeputialis e Platynosomum fastosum & - & - & 2 & 0,012 \\
\hline Ancylostoma spp e Dipylidium caninum & - & - & 1 & 0,006 \\
\hline Ancylostoma spp e Platynosomum fastosum & - & - & 1 & 0,006 \\
\hline Ancylostoma spp, Entamoeba spp e Toxocara spp & 2 & 0,002 & - & - \\
\hline Toxocara spp, Isospora spp e Giardia spp & 2 & 0,002 & - & - \\
\hline Toxocara spp, Entamoeba spp e Isospora spp & 2 & 0,002 & - & - \\
\hline Ancylostoma spp, Isospora spp e Trichuris vulpis & 1 & 0,001 & - & - \\
\hline Ancylostoma spp, Isospora spp e Toxocara spp & 1 & 0,001 & - & - \\
\hline Ancylostoma spp, Toxocara spp e Trichuris vulpis & 1 & 0,001 & - & - \\
\hline Toxocara spp, Trichuris vulpis e Isospora spp & 1 & 0,001 & - & - \\
\hline Toxocara spp, Dipylidium caninum e Ancylostoma spp & 1 & 0,001 & - & - \\
\hline Giardia spp, Trichomonas spp, Isospora spp & 1 & 0,001 & - & - \\
\hline Entamoeba spp, Giardia spp e Isospora spp & 1 & 0,001 & - & - \\
\hline Giardia spp, Entamoeba spp e Trichomonas spp & 1 & 0,001 & - & - \\
\hline Ancylostoma spp, Taenia spp e P. fastosum & - & - & 1 & 0,006 \\
\hline H. hammondi, Isospora spp e Ancylostoma spp & - & - & 1 & 0,006 \\
\hline Toxocara spp, Isospora spp, Entamoeba spp e Giardia spp & 1 & 0,001 & - & - \\
\hline
\end{tabular}

Fonte:Elaboração dos autores.

Giardia spp., na maioria das vezes, não diagnóstico e facilita a propagação dos cistos no causa sintomas em cães e gatos, o que dificulta o meio ambiente, o homem pode se infectar ao ingerir 
água, alimentos ou até pela contaminação das mãos (ROBERTSON et al., 2000; CAVALINI; ZAPPA, 2011). A frequência de Giardia spp. observada neste estudo de $8,65 \%$ nos cães é semelhante a obtida por Labruna et al. (2006) e Funada et al. (2007) que encontraram 8,42\% em 95 cães de uma área urbana de Monte Negro - RO e 8,49\% de um levantamento com 1.755 cães atendidos no Hospital Veterinário da Universidade de São Paulo (USP), respectivamente. Já em gatos, no presente trabalho, houve uma ocorrência de $10,85 \%$, resultado inferior ao descrito por Lima et al. (2006) que estimaram a frequência de endoparasitos através de 85 amostras de gatos da cidade de Goiânia e encontraram 11,8\% de animais positivos. Existe uma maior frequência em animais domiciliados, quando comparados com errantes (RAGOZO et al., 2002; ALVES; GOMES; SILVA, 2005).

O hábito de animais frequentarem locais públicos como praças, parques e praias, ambientes favoráveis ao desenvolvimento de parasitos como Ancylostoma spp. e Toxocara spp., constitui um problema em saúde pública, uma vez que cães e gatos infectados podem contaminar esses locais e transmitir para humanos, causando Larva Migrans Cutânea e Visceral, respectivamente (COSTA-CRUZ; NUNES; BUSO, 1994; SCAINI et al., 2003; SANTARÉM; GIUFFRIDA; ZANIN, 2004; FRANCISCO et al., 2008). No levantamento foi observada uma frequência para Ancylostoma spp. de 6,51\% em cães e $9,26 \%$ em gatos. Resultado similar foi observado em um trabalho também realizado na cidade de Londrina com 150 cães que apresentavam diarreia, destes 7,3\% eram positivos para Ancylostoma spp. (SANTOS et al., 2007). Ragozo et al. (2002) coletaram amostras de fezes de 138 gatos capturados das ruas de São Paulo e Guarulhos e notaram que $8,70 \%$ dos animais tinham o parasito nas fezes.

A frequência de Toxocara spp. foi 6,03\% em cães e $0,79 \%$ em gatos. Nos trabalhos reportados houve uma variação de $0,8 \%$, de 121 amostras de fezes de animais de uma praia em Porto Alegre RS (MATESCO et al., 2006), a 71,43\%, da fauna helmíntica de 28 cães necropsiados de Araraquara, Cosmorama, Jaboticabal, Pontal, Sertãozinho e Taiúva, Estado de São Paulo (SILVA et al., 2001). Nos felinos a flutuação foi de 3,33\%, de 30 amostras de fezes de gatos de Curitiba - PR (TESSEROLLI; FAYZANO; AGOTTANI, 2005), a 34,22\%, de 187 amostras fecais analisadas no Laboratório de Doenças Parasitárias da USP no período de 19911995 (GENNARI et al., 1999).

Embora o gênero Entamoeba seja considerado, muitas vezes, composto por parasitos não patogênicos, é importante que seja feito o diagnóstico para diferenciação das espécies, pois existe tanto Entamoeba coli, que são comensais, colonizadores dos intestinos do homem e de animais, quanto a $E$. histolitica, ameba de importância médico veterinária (CHIEFFI; GRYSCHEK; AMATO NETO, 2000; NEVES, 2005). Foi encontrada, neste estudo, uma frequência de 4,15\% nos cães e de 4,76\% nos gatos, parasitados por Entamoeba spp. Santos et al. (2007), obtiveram uma frequência de $6,7 \%$ nos animais com diarreia atendidos no Hospital Veterinário da Universidade Estadual de Londrina. Os gatos apresentaram uma frequência superior ao descrito por Brener et al. (2005) que encontrou 2,5\% de amostras positivas em fezes analisadas na rotina do Laboratório de Parasitologia do Departamento de Microbiologia e Parasitologia da Universidade Federal Fluminense (UFF) no período de 1999 a 2002.

Diante dos resultados expostos, deve-se considerar o fato de que a maioria dos animais é domiciliada, ou seja, tem maior contato com o homem, podendo ser o veiculador de importantes zoonoses.

Os protozoários Isospora spp. e Giardia spp. foram os parasitas mais frequentemente observados nos cães e gatos. Isso se deve provavelmente pela administração de antiparasitários sem a devida orientação de um médico veterinário.

Assim, para que ocorra uma redução na frequência de parasitos gastrintestinais, medidas profiláticas devem ser incorporadas à rotina $\mathrm{e}$ 
aos hábitos humanos. Além disso, o manejo e tratamento das parasitoses gastrintestinais precisam ser realizados com duração e intervalo adequados, de preferência com diagnóstico prévio para que a intervenção terapêutica seja eficaz. A redução da carga parasitária dos animais e, consequentemente, do ambiente diminui a exposição dos humanos a importantes zoonoses.

\section{Referências}

ALVES, O. F.; GOMES, A. G.; SILVA, A. C. Ocorrência de enteroparasitoses em cães do Município de Goiânia, Goiás: Comparação de técnicas de diagnóstico. Ciência Animal Brasileira, Goiânia, v. 6, n. 2, p. 127-133, 2005.

BRENER, B.; LISBOA, L.; MATTOS, D. P. B. G.; ARASHIRO, E. K. N.; MILLAR, P. R.; SUDRÉ, A. P.; DUQUE, V. Frequência de enteroparasitas em amostras fecais de cães e gatos dos municípios do Rio de Janeiro e Niterói. Revista Brasileira de Ciência Veterinária, Rio de Janeiro, v. 12, n. 1-3, p. 102-105, 2005.

CAMPOS FILHO, P. C.; BARROS, L. M.; CAMPOS, J. O.; BRAGA, V. B.; CAZORLA, I. M.; ALBUQUERQUE, G. R.; CARVALHO, S. M. S. Parasitas zoonóticos em fezes de cães em praças públicas do município de Itabuna, Bahia, Brasil. Revista Brasileira de Parasitologia Veterinária, Jaboticabal, v. 17, n. 4, p. 206-209, 2008.

CAVALINI, P. P.; ZAPPA, V. Giardíase felina - revisão de literatura. Revista Científica Eletrônica de Medicina Veterinária, São Paulo, Ano IX, n. 16, p. 1-18, jan. 2011.

CHIEFFI, P. P.; GRYSCHEK, R. C. B.; AMATO NETO, V. Diagnóstico e tratamento de parasitoses intestinais. Revista Brasileira de Clínica e Terapêutica, São Paulo, v. 25 , n. 5, p. 163-170, 2000.

COSTA-CRUZ, J. M.; NUNES, R. S.; BUSO, A. G. Presença de ovos de Toxocara spp em praças públicas da cidade de Uberlândia, Minas Gerais, Brasil. Revista do Instituto de Medicina Tropical de São Paulo, São Paulo, v. 36, n. 1, p. 39-42, 1994.

DIMAS, W. C.; SEBADELHE, D.; RODRIGUES, A. C. Incidência de parasitos gastrointestinais em cães no bairro Jardim Paraíso da cidade de São Vicente, São Paulo, Brasil. In: REUNIÃO ANUAL DO INSTITUTO BIOLÓGICO, 2.; CONGRESSO DE INICIAÇÃO CIENTÍFICA EM CIÊNCIAS AGRÁRIAS, BIOLÓGICAS E AMBIENTAIS, 17., 2004, São Paulo. Anais... São Paulo: Arquivos do Instituto Biológico, 2004, v. 71, p. 68.
FAUST, E. C.; SAWITZ, W.; TOBIE, J. ODOM, V.; PERES, C.; LINCICOME, D. R. Comparative efficiency of various technics for the diagnosis of protozoa and helminth in feces. International Journal of Parasitology, Oxford, v. 25, p. 241-262, 1939.

FRANCISCO, M. M. S.; SILVA, R. C.; FIGUEIREDO, D. L. V.; SOUZA, J. N.; RAMALHO, P. C. D.; CAETANO, A. L. Prevalência de ovos e larvas de Ancylostoma spp. e Toxocara spp. em praças públicas da cidade de Anápolis - GO. Ensaio e Ciências: Ciências Biológicas, Agrárias e da Saúde, São Paulo, v. 12, n. 1, p. 131-137, 2008.

FUNADA, M. R.; PENA, H. F. J.; SOARES, R. M.; AMAKU, M.; GENNARI, S. M. Frequência de parasitos gastrintestinais em cães e gatos atendidos em hospitalescola veterinário da cidade de São Paulo. Arquivo Brasileiro de Medicina Veterinária e Zootecnia, Belo Horizonte, v. 59, n. 5, p.1338-1340, 2007.

GENNARI, S. M.; KASAI, N.; PENA, H. F. J.; CORTEZ, A. Ocorrência de protozoários e helmintos em amostras de fezes de cães e gatos da cidade de São Paulo. Brazilian Journal of Veterinary Research and Animal Science, São Paulo, v. 36, n. 2, p. 87-91, 1999.

HOFFMANN, R. P. Exame parasitológico de fezes. In:_. Diagnóstico de parasitismo veterinário. Porto Alegre: Sulina, 1987, p. 26-32.

HOFFMANN, W. A.; PONS, J. A.; JANER, J. L. Sedimentation concentration method in schistosomiasis, Puerto Rico. Journal of Public Health, Oxford, v. 9, p. 283-298, 1934.

LABRUNA, M. B.; PENA, H. F. J.; SOUZA, S. L. P.; PINTER, A.; SILVA, J. C. R.; RAGOZO, A. M. A.; CAMARGO, L. M. A.; GENNARI, S. M. Prevalência de endoparasitas em cães da área urbana do município de Monte Negro, Rondônia. Arquivos do Instituto Biológico, São Paulo, v. 73, p. 183-193, 2006.

LANGONI, H. Zoonoses and human beings. The Journal of Venomous Animals and Toxins including Tropical Diseases, Botucatu, v. 10, n. 2, p. 111-111, 2004.

LEITE, L. C.; CÍRIO, S. M.; NAVARRO-SILVA, M. A.; ZADOROSNEI, A. C. B.; LUZ, E. MARIONI, L. P.; LEITE, S. C.; LUNELLI, D. Ocorrência de endoparasitas em amostras de fezes de cães (Canis familiaris) da região metropolitana de Curitiba, Paraná - Brasil. Revista Estudo de Biologia, v. 29, n. 68-69, p. 319-326, 2007.

LIMA, F. G.; AMARAL, A. V. C.; OLIVEIRA, R.; ALVES, E.; SILVA, E. B.; TASSARA, N.; FREITAS, P. H. O.; BARBOSA, V. T. Frequência de enteroparasitas em gatos no município de Goiania - Goiás, no ano de 2004. Enciclopédia Biosfera, 2006. n. 2, 4 p. Disponível em: <http://www.conhecer.org.br/ enciclop/2006/FREQUENCIA.pdf>. Acesso em: 23 jul. 2012. 
MACHADO, J. A. C.; ROCHA, J. R.; SANTOS, L. M.; PICCININ, A. Terapia assistida por animais (TAA). Revista Científica Eletrônica de Medicina Veterinária, Garça, Ano VI, n. 10, p. 1-7, 2008.

MATESCO, V. C.; MENTZ, M. B.; ROOT, M. B., SILVEIRA, C. O. Contaminação sazonal por ovos de helmintos na praia de Ipanema, em Porto Alegre, Rio Grande do Sul, Brasil. Revista de Patologia Tropical, São Paulo, v. 35, p. 135-141, 2006.

NEVES, D. P. Parasitologia humana. 11. ed. São Paulo: Editora Atheneu, 2005. 494 p.

OLIVEIRA, S. F. O.; MELO, D. P. G.; FERNANDES, P. R.; SCHULZE, C. M. B.; GUIMARÃES, M. S.; SILVA, Q. C. Ocorrência de helmintos gastrintestinais em cães errantes da cidade de Goiânia - Goiás. Revista de Patologia Tropical, São Paulo, v. 38, n. 4, p. 279-283, 2009.

PEREIRA, A. D.; DAMIN, J.; LIMA, L. M.; UIANO, R. W. Artigo de Revisão - Isospora belli: aspectos clínicos e diagnóstico laboratorial. Revista Brasileira de Análises Clínicas, Rio de Janeiro, v. 41, n. 4, p. 283-286, 2009.

RAGOZO, A. M. A.; SILVA, J. C. R.; CARAVIERI, R.; AMAJONER, V. R.; MAGNABOSCO, C.; GENNARI, S. M. Ocorrência de parasitos gastrintestinais em fezes de gatos das cidades de São Paulo e Guarulhos. Brazilian Journal of Veterinary Research and Animal Science, São Paulo, v. 39, n. 5, p. 244-246, 2002.

ROBERTSON, I. D.; IRWIN, P. J.; LYMBERY, A. J.; THOMPSON, R. C. A. The role of companion animals in the emergence of parasitic zoonoses. International Journal for Parasitology, Oxford, v. 30, n. 2, p. 13691377, 2000.

SANTARÉM, V. A.; GIUFFRIDA, R.; ZANIN, G. A. Larva migrans cutânea: ocorrência de casos humanos e identificação de larvas de Ancylostoma spp em parque público do município de Taciba, São Paulo. Revista da Sociedade Brasileira de Medicina Tropical, Uberaba, v. 37, n. 2, p. 179-181, 2004.

SANTOS, F. A. G.; YAMAMURA, M. H.; VIDOTTO, O.; CAMARGO, P. L. Ocorrência de parasitos gastrintestinais em cães (Canis familiaris) com diarreia aguda oriundos da região metropolitana de Londrina, Estado do Paraná, Brasil. Semina: Ciências Agrárias, Londrina, v. 28, n. 2, p. 257-268, 2007.

SCAINI, C. J.; TOLEDO, R. N.; LOVATEL, R.; DIONELLO, M. A.; GATTI, F. A.; SUSIN, L.; SIGNORINI, V. R. M. Contaminação ambiental por ovos e larvas de helmintos em fezes de cães na área central do Balneário Cassino, Rio Grande do Sul. Revista da Sociedade Brasileira de Medicina Tropical, Uberaba, v.
36, n. 5, p. 617-619, 2003.

SCHANTZ, P. M. Parasitic zoonoses in perspective. International Journal for Parasitology, Oxford, v. 21, n. 2, p. 161-70, 1991.

SILVA, H. C.; CASTAGNOLLI, K. C.; SILVEIRA, D. M.; COSTA, G. H. N.; GOMES, R. A.; NASCIMENTO, A. A. Fauna helmíntica de cães e gatos provenientes de alguns municípios do Estado de São Paulo. Semina: Ciências Agrárias, Londrina, v. 22, n. 1, p. 67-71, 2001.

SILVA, A. S.; CEOLIN, L. V.; CARGNELUTTI, J. F.; PESSOA, G. A.; OLIVEIRA, C. B.; QUINTAL, A. P. N.; MONTEIRO, S. G. Prevalência de parasitismo em cães domiciliados num bairro de Santa Maria - RS. Saúde, Santa Maria, v. 33, n. 1, p. 27-31, 2007.

SILVA, A. S.; MAURERI, C. G.; GASPPERI, D. D.; PESSOA, G. A.; ZANETTE, R. A.; ANTONOW, R. R.; VOGEL, F. S. F.; SANGIONI, L. A.; MONTEIRO, S. G. Protozoários em cães de canis de Santa Maria - RS. Revista da FZVA, Uruguaiana, v. 15, n. 1, p. 191-199. 2008.

TESSEROLLI, G. L.; FAYZANO, L.; AGOTTANI, J. V. B. Ocorrência de parasitas gastrintestinais em fezes de cães e gatos, Curitiba-PR. Revista Acadêmica, Curitiba, v. 3, n. 4, p. 31-34, 2005.

TORRICO, K. J.; SANTOS, K. R.; MARTINS, T. F.; PAZ E SILVA, F. M.; TAKAHIRA, R. K.; LOPES, R. S. Ocorrência de parasitas gastrintestinais em cães e gatos na rotina do laboratório de enfermidades parasitárias da FMVZ/UNESP-Botucatu, SPP. Revista Brasileira de Parasitologia Veterinária, Jaboticabal, v. 17, p. 182-183, 2008. Suplemento 1.

URQUHART, G. M.; ARMOUR, J.; DUNN, A. M.; JENNINGS, F. W. Parasitologia veterinária. 2. ed. Rio de Janeiro: Guanabara Koogan, 1998.

VASCONCELLOS, M. C.; BARROS, J. S. L.; OLIVEIRA, C. S. Parasitas gastrointestinais em cães institucionalizados no Rio de Janeiro, RJ. Revista de Saúde Pública, São Paulo, v. 40, n. 2, p. 321-323, 2006.

VASCONCELOS, M. G. C.; TALON, D. D. B.; SILVA JÚNIOR, C. A.; NEVES, M. F.; SACCO, S. R. Isosporose nos animais domésticos. Revista Científica Eletrônica de Medicina Veterinária, Garça, v. 6, n. 10, p. 1-7, 2008.

WILLIS, H. H. A simple levitation method for the detection of hookworm ova. Medical Journal of Australia, North Sidney, v. 8, n. 3, p. 375-376, 1921.

XAVIER, G. A. Prevalência de endoparasitos em cães de companhia em Pelotas-RS e risco zoonótico. 2006. Monografia (Conclusão do Curso de Ciências Biológicas) - Universidade Federal de Pelotas, Pelotas. 